Supplement of The Cryosphere, 12, 81-94, 2018 https://doi.org/10.5194/tc-12-81-2018-supplement (C) Author(s) 2018. This work is distributed under the Creative Commons Attribution 3.0 License.

(c) (1)

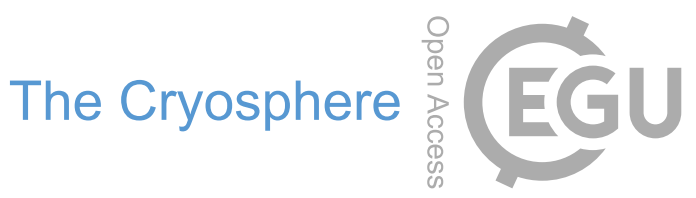

Supplement of

\title{
The Greater Caucasus Glacier Inventory (Russia, Georgia and Azerbai- jan)
}

\section{Levan G. Tielidze and Roger D. Wheate}

Correspondence to: Levan G. Tielidze (levan.tielidze@tsu.ge)

The copyright of individual parts of the supplement might differ from the CC BY 3.0 License. 
Table S1. The Greater Caucasus glacier number and area change in 1960-1986, 1986-2014 and 1960-2014 by individual river basins.

\begin{tabular}{|c|c|c|c|c|c|c|c|c|c|c|c|c|}
\hline \multirow{3}{*}{ Main river basin } & \multirow{3}{*}{$\begin{array}{c}\text { Tributary river } \\
\text { basin }\end{array}$} & \multirow{2}{*}{\multicolumn{2}{|c|}{$\begin{array}{c}\text { USSR Catalogue } \\
1967-1978\end{array}$}} & \multirow{2}{*}{\multicolumn{2}{|c|}{ Topographic maps 1960}} & \multirow{2}{*}{\multicolumn{2}{|c|}{ Landsat 5, 1985-1987 }} & \multirow{2}{*}{\multicolumn{2}{|c|}{$\begin{array}{c}\text { Landsat 8, 2013-2016 } \\
\text { and ASTER } 2014\end{array}$}} & \multicolumn{3}{|c|}{ Area change } \\
\hline & & & & & & & & & & \multirow{2}{*}{$\begin{array}{r}1960- \\
1986 \\
\% \mathrm{yr}^{-1}\end{array}$} & \multirow{2}{*}{$\begin{array}{r}1986- \\
2014 \\
\% \mathrm{yr}^{-1}\end{array}$} & \multirow{2}{*}{$\begin{array}{r}1960- \\
2014 \\
\% \mathrm{yr}^{-1}\end{array}$} \\
\hline & & Number & Area $\mathrm{km}^{2}$ & Number & Area km² & Number & Area km² & Number & Area $\mathrm{km}^{2}$ & & & \\
\hline Mzimta & & 7 & 1.7 & 13 & $2.4 \pm 0.2$ & 13 & $2.3 \pm 0.2$ & 13 & $1.9 \pm 0.2$ & -0.16 & -0.62 & -0.38 \\
\hline \multirow{9}{*}{ Kuban } & Belaya & 29 & 7.6 & 39 & $7.6 \pm 0.4$ & 45 & $8.3 \pm 0.6$ & 50 & $6.6 \pm 0.4$ & +0.35 & -0.73 & -0.24 \\
\hline & Malaya Laba & 27 & 9.4 & 39 & $11.2 \pm 0.6$ & 45 & $8.4 \pm 0.6$ & 39 & $7.5 \pm 0.6$ & -0.96 & -0.38 & -0.61 \\
\hline & Bolshaya Laba & 21 & 5.8 & 30 & $7.1 \pm 0.4$ & 30 & $6.7 \pm 0.4$ & 29 & $4.5 \pm 0.2$ & -0.21 & -1.17 & -0.67 \\
\hline & Bolshoy Zelenchuk & 56 & 30.7 & 58 & $32.4 \pm 1.6$ & 65 & $33.8 \pm 1.8$ & 74 & $25.6 \pm 1.6$ & +0.16 & -0.86 & -0.38 \\
\hline & Maliy Zelenchuk & 28 & 26.3 & 37 & $30.3 \pm 1.2$ & 41 & $29.8 \pm 1.0$ & 46 & $24.6 \pm 1.2$ & -0.06 & -0.67 & -0.34 \\
\hline & Teberda & 85 & 61.0 & 99 & $63.6 \pm 2.8$ & 103 & $62.1 \pm 2.6$ & 100 & $53.7 \pm 2.6$ & -0.09 & -0.48 & -0.28 \\
\hline & Daut & 16 & 5.5 & 19 & $7.2 \pm 0.4$ & 22 & $6.1 \pm 0.4$ & 24 & $4.8 \pm 0.4$ & -0.58 & -0.76 & -0.61 \\
\hline & Uchkulan & 58 & 20.8 & 64 & $27.3 \pm 1.4$ & 65 & $22.7 \pm 1.4$ & 71 & $19.2 \pm 1.4$ & -0.64 & -0.55 & -0.54 \\
\hline & Ullukam & 88 & 52.9 & 107 & $65.2 \pm 3.2$ & 105 & $51.0 \pm 2.6$ & 95 & $42.5 \pm 2.2$ & -0.83 & -0.59 & -0.64 \\
\hline Malka & & 10 & 56.5 & 10 & $64.0 \pm 1.0$ & 11 & $57.5 \pm 0.8$ & 12 & $52.2 \pm 0.8$ & -0.39 & -0.32 & -0.34 \\
\hline Baksan & & 156 & 132.7 & 170 & $187.0 \pm 7.0$ & 162 & $169.8 \pm 6.4$ & 156 & $146.3 \pm 6.0$ & -0.35 & -0.49 & -0.40 \\
\hline \multirow{3}{*}{ Chegem } & Bashil-Auzusu & 28 & 26.9 & 39 & $34.6 \pm 1.4$ & 31 & $30.1 \pm 1.2$ & 30 & $25.3 \pm 1.0$ & -0.50 & -0.56 & -0.49 \\
\hline & Gara-Auzusu & 28 & 28.0 & 21 & $30.1 \pm 1.0$ & 19 & $29.3 \pm 1.2$ & 17 & $24.7 \pm 1.0$ & -0.10 & -0.56 & -0.33 \\
\hline & Bulungu & 9 & 3.2 & 8 & $6.6 \pm 0.2$ & 10 & $4.0 \pm 0.2$ & 9 & $2.9 \pm 0.2$ & -1.51 & -0.98 & -1.03 \\
\hline \multirow{3}{*}{ Cherek } & Cherek-Bezingskiy & 85 & 76.3 & 48 & $82.4 \pm 2.8$ & 40 & $81.1 \pm 2.6$ & 48 & $73.8 \pm 2.6$ & -0.06 & -0.32 & -0.19 \\
\hline & Cherek-Balkarskiy & 80 & 107.0 & 78 & $115.0 \pm 3.8$ & 79 & $113.0 \pm 3.8$ & 86 & $96.8 \pm 3.6$ & -0.06 & -0.51 & -0.29 \\
\hline & Psigansy & 17 & 15.0 & 13 & $15.2 \pm 0.6$ & 12 & $15.6 \pm 0.6$ & 13 & $12.8 \pm 0.6$ & +0.10 & -0.64 & -0.29 \\
\hline \multirow{5}{*}{ Urukh } & Khanzidon & 13 & 4.7 & 14 & $8.5 \pm 0.4$ & 15 & $8.4 \pm 0.4$ & 12 & $6.3 \pm 0.4$ & -0.04 & -0.89 & -0.47 \\
\hline & Biliagikom & 5 & 2.0 & 6 & $2.5 \pm 0.1$ & 4 & $2.1 \pm 0.2$ & 5 & $1.0 \pm 0.1$ & -0.61 & -1.87 & -1.11 \\
\hline & Urukh headwaters & 34 & 23.0 & 35 & $31.1 \pm 1.2$ & 33 & $30.3 \pm 1.4$ & 35 & $25.3 \pm 1.2$ & -0.09 & -0.58 & -0.34 \\
\hline & Karaugom & 27 & 39.6 & 21 & $48.4 \pm 1.4$ & 23 & $45.7 \pm 1.4$ & 31 & $41.7 \pm 1.4$ & -0.21 & -0.31 & -0.25 \\
\hline & Aigamuga & 26 & 13.4 & 14 & $24.0 \pm 1.0$ & 17 & $17.4 \pm 0.6$ & 20 & $14.7 \pm 0.8$ & -1.05 & -0.55 & -0.71 \\
\hline \multirow{2}{*}{ Ardon } & Tseyadon & 29 & 15.0 & 22 & $21.4 \pm 0.8$ & 18 & $19.2 \pm 0.6$ & 20 & $16.8 \pm 0.6$ & -0.39 & -0.44 & -0.39 \\
\hline & Sidan & 1 & 0.05 & 1 & $0.08 \pm 0.01$ & 1 & $0.07 \pm 0.01$ & 1 & $0.03 \pm 0.04$ & -0.48 & -2.04 & -1.15 \\
\hline
\end{tabular}




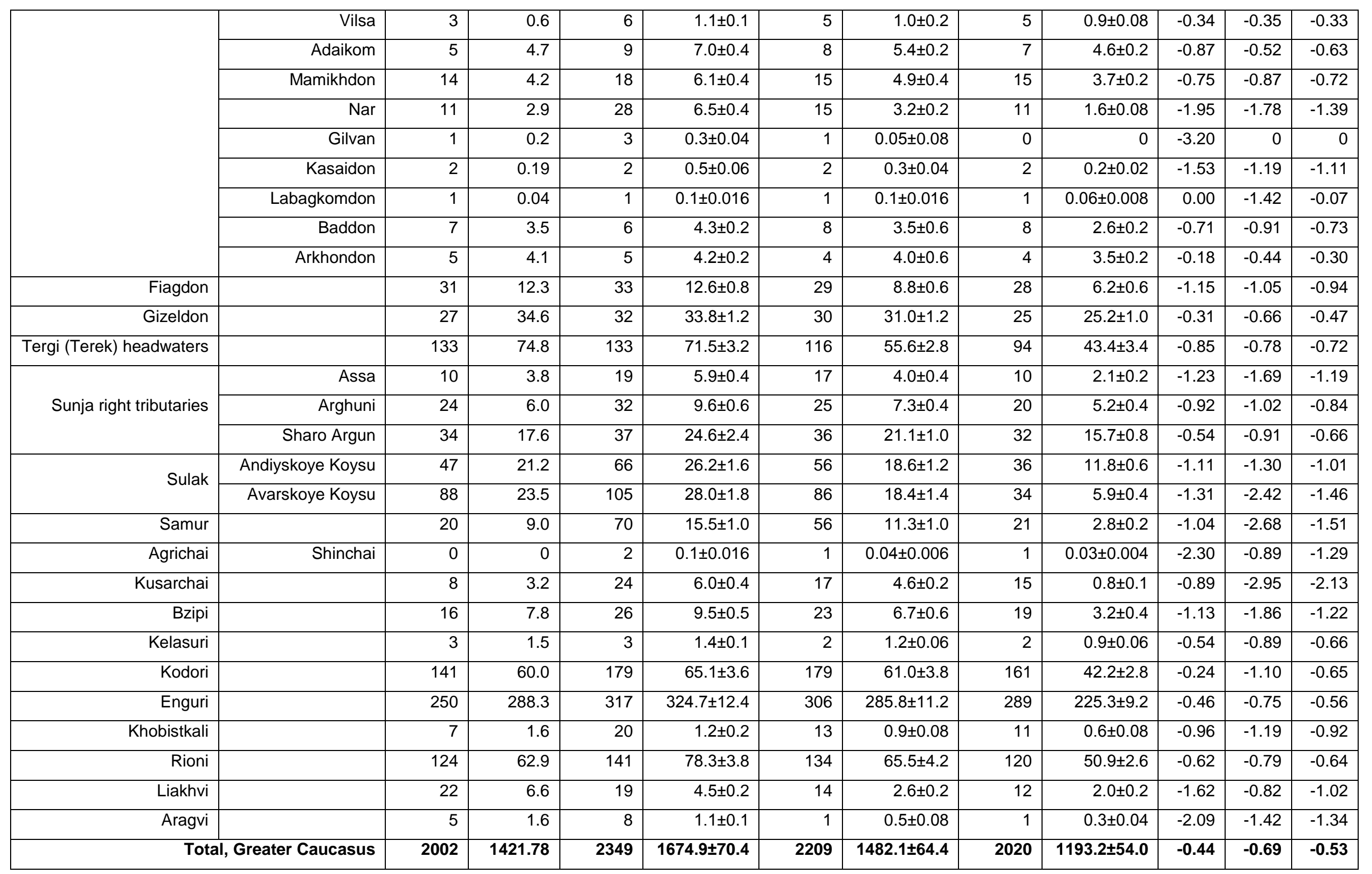


Table S2. The Greater Caucasus glacier number and area change in 1960-1986, 1986-2014 and 1960-2014 by countries.

\begin{tabular}{|c|c|c|c|c|c|c|c|c|c|}
\hline \multirow[t]{2}{*}{ Countries } & \multicolumn{2}{|c|}{ Topographic maps 1960} & \multicolumn{2}{|c|}{ Landsat 5, 1985-1987 } & \multicolumn{2}{|c|}{$\begin{array}{l}\text { Landsat 8, 2013-2016 } \\
\text { and ASTER } 2014\end{array}$} & \multirow{2}{*}{$\begin{array}{c}\text { Decrease } \\
1960-1986 \\
\% \mathrm{yr}^{-1}\end{array}$} & \multirow{2}{*}{$\begin{array}{c}\text { Decrease } \\
1986-2014 \\
\% \mathrm{yr}^{-1}\end{array}$} & \multirow{2}{*}{$\begin{array}{c}\text { Decrease } \\
1960-2014 \\
\% \mathrm{yr}^{-1}\end{array}$} \\
\hline & Number & Area km² & Number & Area km² & Number & Area km² & & & \\
\hline Russia & 1417 & $099.1 \pm 44.2$ & 1367 & $992.4 \pm 41.4$ & 1275 & $822.2 \pm 35.8$ & 0.37 & 0.61 & 0.46 \\
\hline Georgia & 899 & $568.5 \pm 25.0$ & 817 & $484.0 \pm 22.4$ & 725 & $369.8 \pm 17.4$ & 0.57 & 0.84 & $\overline{0.64}$ \\
\hline Azerbaijan & 33 & $7.3 \pm 0.6$ & 25 & $5.7 \pm 0.6$ & 20 & $1.2 \pm 0.2$ & 0.84 & 2.81 & 1.54 \\
\hline
\end{tabular}

Table S3. Elbrus glacier number and area change in 1960-1986, 1986-2014 and 1960-2014. All glaciers are shown in Fig. S1.

\begin{tabular}{|c|c|c|c|c|c|c|c|c|}
\hline \multicolumn{3}{|c|}{ Elbrus glaciers } & $\begin{array}{c}\text { Topographic } \\
\text { mans } 1960\end{array}$ & $\begin{array}{l}\text { Landsat 5, } \\
06 / 08 / 1986\end{array}$ & $\begin{array}{c}\text { Landsat } 8, \\
03 / 08 / 2014 /\end{array}$ & \multicolumn{3}{|c|}{ Area change } \\
\hline \# & Name & WGI ID & Area $\mathrm{km}^{2}$ & Area $\mathrm{km}^{2}$ & Area $\mathrm{km}^{2}$ & $\begin{array}{r}1960-1986 \\
\% y^{-1}\end{array}$ & $\begin{array}{r}1986-2014 \\
\% y^{-1}\end{array}$ & $\begin{array}{r}1960-2014 \\
\% y^{-1}\end{array}$ \\
\hline 1 & Ulluchiran & SU4G08005001 & $12.87 \pm 0.26$ & $13.12 \pm 0.16$ & $12.68 \pm 0.18$ & +0.07 & -0.11 & -0.02 \\
\hline 2 & Karachaul & SU4G08005002 & $6.20 \pm 0.16$ & $6.22 \pm 0.12$ & $5.96 \pm 0.14$ & +0.01 & -0.14 & -0.07 \\
\hline 3 & Ullukol & SU4G08005003 & $6.42 \pm 0.16$ & $5.81 \pm 0.12$ & $5.45 \pm 0.12$ & -0.36 & -0.22 & -0.28 \\
\hline 4 & $565 a$ & SU4G08005004 & $0.97 \pm 0.04$ & $0.54 \pm 0.04$ & $0.13 \pm 0.02$ & -1.70 & -2.71 & -1.60 \\
\hline 5 & Mikelchiran & SU4G08005005 & $7.74 \pm 0.12$ & $7.09 \pm 0.12$ & $6.84 \pm 0.12$ & -0.32 & -0.12 & -0.21 \\
\hline 6 & Dzhikiugankez & SU4G08005006 & $28.41 \pm 0.24$ & $24.0 \pm 0.3$ & $20.68 \pm 0.32$ & -0.59 & -0.53 & -0.50 \\
\hline 7 & Irikchat & SU4G08005018 & $1.56 \pm 0.02$ & $1.29 \pm 0.06$ & $1.09 \pm 0.04$ & -0.66 & -0.55 & -0.55 \\
\hline 8 & Irik & SU4G08005020 & $12.56 \pm 0.28$ & $11.46 \pm 0.22$ & $10.95 \pm 0.22$ & -0.33 & -0.15 & -0.23 \\
\hline 9 & Terskol & SU4G08005026 & $9.83 \pm 0.32$ & $9.78 \pm 0.16$ & $9.45 \pm 0.14$ & -0.01 & -0.12 & -0.07 \\
\hline 10 & Garabashi & SU4G08005027 & $3.13 \pm 0.08$ & $2.75 \pm 0.08$ & $2.45 \pm 0.1$ & -0.46 & -0.38 & -0.40 \\
\hline 11 & Maliy Azau & SU4G08005028 & $10.08 \pm 0.16$ & $10.03 \pm 0.14$ & $9.41 \pm 0.18$ & -0.01 & -0.22 & -0.12 \\
\hline 12 & Bolshoy Azau & SU4G08005029 & $21.26 \pm 0.34$ & $20.47 \pm 0.32$ & $18.20 \pm 0.36$ & -0.14 & -0.39 & -0.26 \\
\hline 13 & 311 & SU4H08004311 & $0.57 \pm 0.04$ & $0.51 \pm 0.04$ & $0.37 \pm 0.02$ & -0.40 & -0.98 & -0.64 \\
\hline 14 & 312 & SU4H08004312 & $0.25 \pm 0.02$ & $0.33 \pm 0.02$ & $0.26 \pm 0.02$ & +1.23 & -0.75 & -0.07 \\
\hline 15 & Ullukam & SU4H08004313 & $0.56 \pm 0.04$ & $0.72 \pm 0.04$ & $0.67 \pm 0.04$ & +1.09 & -0.24 & -0.36 \\
\hline$\overline{16}$ & 313 & SU4H08004313 & $1.08 \pm 0.06$ & $0.98 \pm 0.06$ & $0.98 \pm 0.06$ & -0.35 & 0 & -0.17 \\
\hline 17 & 317 & SU4H08004317 & $0.74 \pm 0.02$ & $0.76 \pm 0.04$ & $0.76 \pm 0.04$ & +0.10 & 0 & +0.05 \\
\hline 18 & Unnamed $^{*}$ & Unknown & $0.65 \pm 0.04$ & $0.59 \pm 0.02$ & $0.52 \pm 0.04$ & -0.35 & -0.42 & -0.37 \\
\hline 19 & Kyukyurtlyu & SU4H08004318 & $5.69 \pm 0.2$ & $5.61 \pm 0.12$ & $5.54 \pm 0.14$ & -0.05 & -0.04 & -0.04 \\
\hline 20 & 319 & SU4H08004319 & $1.54 \pm 0.06$ & $0.98 \pm 0.06$ & $0.94 \pm 0.04$ & -1.39 & -0.14 & -0.72 \\
\hline 21 & Bityuktyube & SU4H08004320 & $2.40 \pm 0.1$ & $1.74 \pm 0.08$ & $1.65 \pm 0.06$ & -1.05 & -0.18 & -0.57 \\
\hline 22 & 321 & SU4H08004321 & $0.38 \pm 0.04$ & $0.21 \pm 0.02$ & $0.08 \pm 0.012$ & -1.72 & -2.21 & -1.46 \\
\hline \multicolumn{3}{|c|}{ Total } & $134.89 \pm 2.84$ & $124.99 \pm 2.74$ & $115.06 \pm 2.68$ & -0.28 & -0.28 & -0.27 \\
\hline
\end{tabular}

\footnotetext{
* Omitted in WGI database
} 


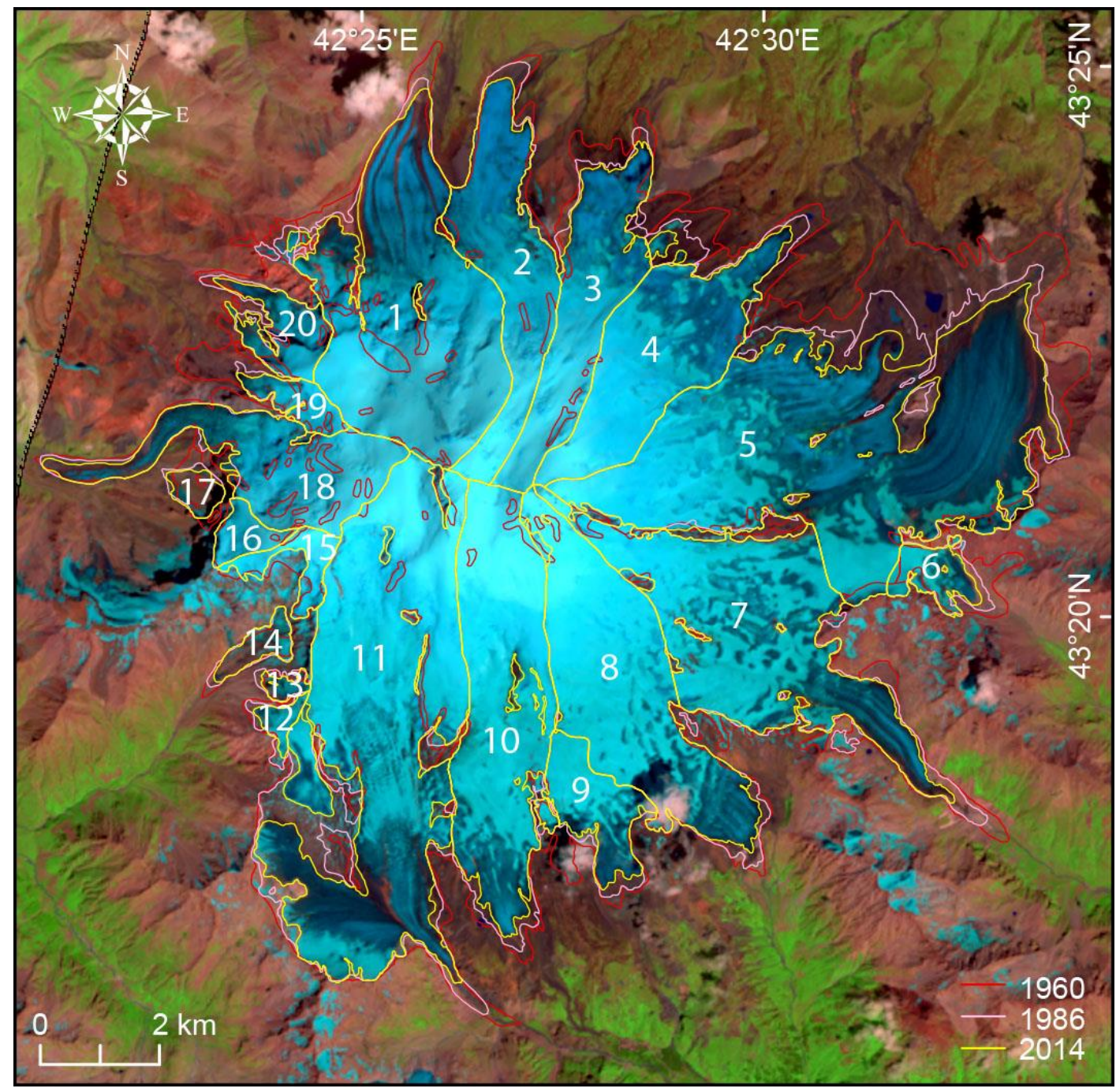

Figure S1. Changes in glacierized area of Elbrus between 1960, 1986 and 2014.

See Table S3 for the change statistics of individual glaciers. The 03 August 2014 Landsat 8 image is used as background. 
Table S4. Kazbegi-Jimara massif glacier number and area change in 1960-1986, 1986-2014 and 1960-2014. All glaciers are shown in Fig. S2.

\begin{tabular}{|c|c|c|c|c|c|c|c|c|}
\hline \multicolumn{3}{|c|}{ Kazbegi-Jimara massif glaciers } & \multirow{2}{*}{$\begin{array}{l}\begin{array}{c}\text { Topographic } \\
\text { maps } 1960\end{array} \\
\text { Area km² }\end{array}$} & \multirow{2}{*}{$\begin{array}{r}\text { Landsat 5, } \\
06 / 08 / 1986\end{array}$} & \multirow{2}{*}{$\begin{array}{l}\text { Landsat } 8, \\
28 / 08 / 14 /\end{array}$} & \multicolumn{3}{|c|}{ Area change } \\
\hline$\#$ & Name & WGI ID & & & & $\begin{array}{r}1960-1986 \\
\% y^{-1}\end{array}$ & $\begin{array}{r}1986-2014 \\
\% y^{-1}\end{array}$ & $\begin{array}{r}1960-2014 \\
\% \mathrm{yr}^{-1}\end{array}$ \\
\hline 1 & Mydagrabyn & SU4G08010031 & $9.98 \pm 0.22$ & $9.73 \pm 0.24$ & $8.16 \pm 0.24$ & -0.09 & -0.57 & -0.33 \\
\hline 2 & Unnamed* & Unknown & & . & $0.16 \pm 0.02$ & 0 & 0 & 0 \\
\hline 3 & Kolka & SU4G08010039 & $5.06 \pm 0.7$ & $4.28 \pm 0.48$ & $2.51 \pm 0.44$ & -0.59 & -1.47 & -0.91 \\
\hline 4 & Unnamed & Unknown & - & 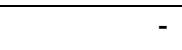 & $0.75 \pm 0.04$ & 0 & 0 & 0 \\
\hline 5 & Unnamed & SU4G08010040 & $0.79 \pm 0.06$ & $0.73 \pm 0.04$ & $0.50 \pm 0.04$ & -0.29 & -1.12 & -0.68 \\
\hline 6 & Maili & SU4G08010041 & $7.29 \pm 0.14$ & $6.75 \pm 0.14$ & $6.57 \pm 0.12$ & -0.28 & -0.09 & -0.18 \\
\hline 7 & Unnamed & Unknown & & & $0.05 \pm 0.008$ & 0 & 0 & 0 \\
\hline 8 & Unnamed & SU4G08010042 & - & $0.67 \pm 0.04$ & $0.44 \pm 0.02$ & 0 & -1.22 & 0 \\
\hline 9 & Chachi & SU4G08011046 & $2.61 \pm 0.06$ & $2.57 \pm 0.08$ & $1.85 \pm 0.08$ & -0.05 & -1.00 & -0.53 \\
\hline 10 & Unnamed & Unknown & $\begin{array}{l}- \\
-\end{array}$ & - & $0.08 \pm 0.012$ & 0 & 0 & 0 \\
\hline 11 & Unnamed & SU4G08011047 & $0.86 \pm 0.06$ & $0.52 \pm 0.04$ & $0.12 \pm 0.02$ & -1.52 & -2.74 & -1.59 \\
\hline 12 & Unnamed & Unknown & - & - & $0.05 \pm 0.008$ & 0 & 0 & 0 \\
\hline 13 & Devdoraki & SU4G08011048 & $7.19 \pm 0.2$ & $6.96 \pm 0.2$ & $4.40 \pm 0.12$ & -0.12 & -1.31 & -0.71 \\
\hline 14 & Unnamed & Unknown & - & - & $1.78 \pm 0.08$ & 0 & 0 & 0 \\
\hline 15 & Abano & SU4G08011049 & $1.96 \pm 0.08$ & $1.49 \pm 0.08$ & $1.33 \pm 0.1$ & -0.92 & -0.38 & -0.60 \\
\hline 16 & Unnamed & Unknown & - & - & $0.03 \pm 0.004$ & 0 & 0 & 0 \\
\hline 17 & Unnamed & Unknown & $0.58 \pm 0.04$ & $0.34 \pm 0.04$ & $0.1 \pm 0.004$ & -1.59 & -2.52 & -1.53 \\
\hline 18 & Gergeti & SU4G08011052 & $6.82 \pm 0.18$ & $6.26 \pm 0.2$ & $5.77 \pm 0.18$ & -0.31 & -0.27 & -0.28 \\
\hline 19 & None & SU4G08011056 & $0.49 \pm 0.04$ & $0.39 \pm 0.02$ & $0.36 \pm 0.02$ & -0.78 & -0.27 & -0.49 \\
\hline 20 & Denkara & SU4G08011057 & $1.33 \pm 0.04$ & $0.93 \pm 0.04$ & $0.31 \pm 0.02$ & -1.15 & -2.38 & -1.42 \\
\hline 21 & Unnamed & Unknown & $0.49 \pm 0.04$ & $0.06 \pm 0.01$ & $0.03 \pm 0.004$ & -3.37 & -1.78 & -1.73 \\
\hline 22 & Unnamed & SU4G08011058 & $0.89 \pm 0.06$ & $0.63 \pm 0.04$ & $0.53 \pm 0.04$ & -1.12 & -0.56 & -0.74 \\
\hline 23 & Unnamed & SU4G08011059 & $1.12 \pm 0.04$ & $0.98 \pm 0.06$ & $0.75 \pm 0.06$ & -0.48 & -0.83 & -0.61 \\
\hline 24 & Mna & SU4G08011060 & $3.25 \pm 0.1$ & $2.89 \pm 0.12$ & $2.59 \pm 0.12$ & -0.42 & -0.37 & -0.37 \\
\hline 25 & Unnamed & SU4G08011061 & $1.57 \pm 0.04$ & $1.30 \pm 0.06$ & $1.44 \pm 0.04$ & -0.66 & +0.38 & -0.15 \\
\hline 26 & Suatisi Eastern & SU4G08011062 & $10.84 \pm 0.2$ & $9.87 \pm 0.24$ & $8.89 \pm 0.18$ & -0.34 & -0.35 & -0.33 \\
\hline 27 & Unnamed & Unknown & - & - & $0.08 \pm 0.012$ & 0 & 0 & 0 \\
\hline 28 & Suatisi Central & SU4G08011063 & $2.62 \pm 0.1$ & $2.32 \pm 0.08$ & $2.07 \pm 0.08$ & -0.44 & -0.38 & -0.39 \\
\hline 29 & Unnamed & Unknown & - & $0.29 \pm 0.04$ & $0.23 \pm 0.02$ & 0 & -0.73 & 0 \\
\hline 30 & Suatisi Western & SU4G08011064 & $2.49 \pm 0.08$ & $2.16 \pm 0.08$ & $1.55 \pm 0.06$ & -0.51 & -1.00 & -0.70 \\
\hline 31 & Unnamed & Unknown & - & - & $0.12 \pm 0.02$ & 0 & 0 & 0 \\
\hline 32 & Unnamed & Unknown & - & 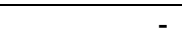 & $0.18 \pm 0.02$ & 0 & 0 & 0 \\
\hline \multicolumn{3}{|c|}{ Total } & $68.23 \pm 2.42$ & $62.12 \pm 2.72$ & $53.78 \pm 2.48$ & -0.34 & -0.47 & -0.39 \\
\hline
\end{tabular}

\footnotetext{
* Omitted in WGI database
} 


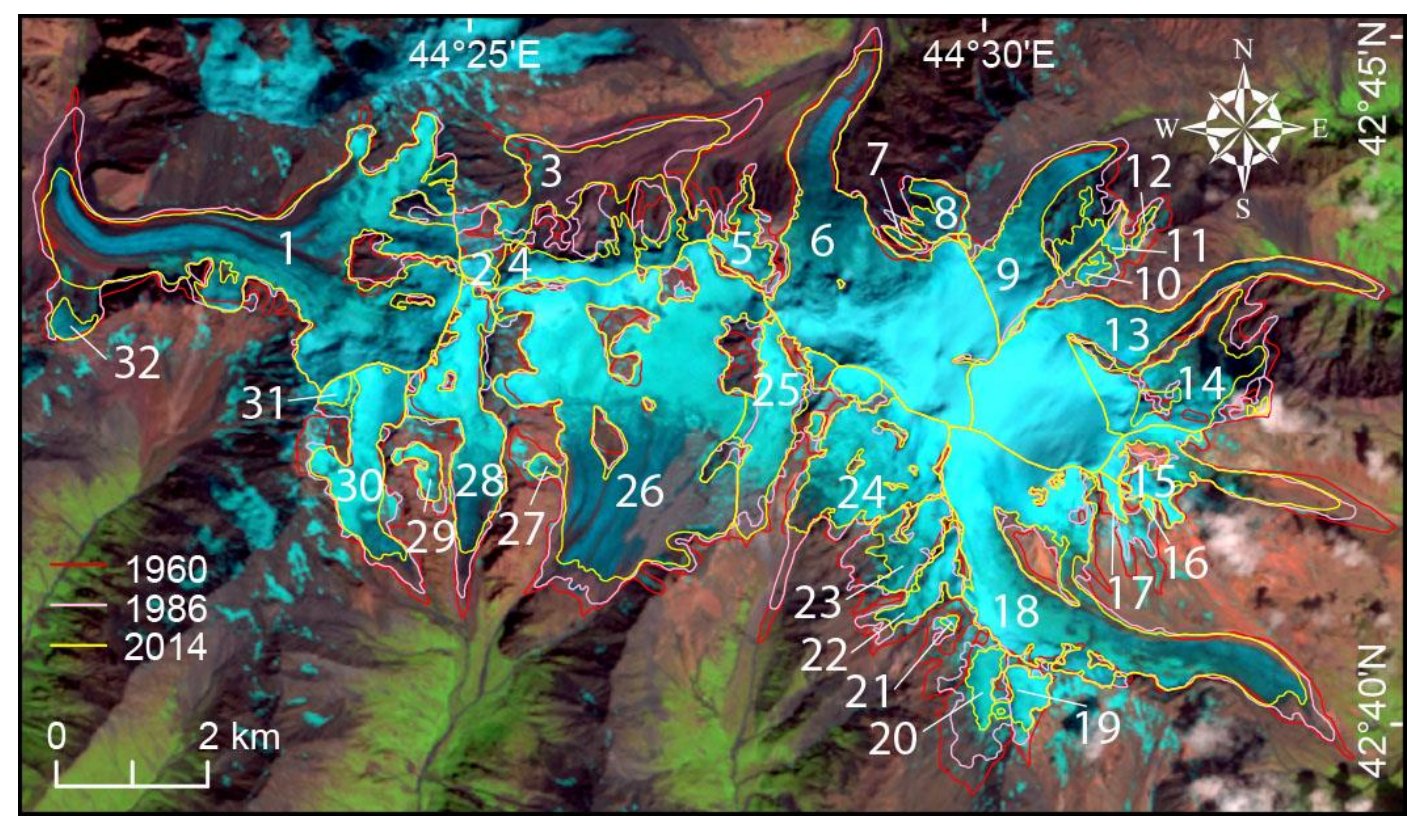

Figure S2. Changes in glacierized area of Kazbegi-Jimara massifs between 1960, 1986 and 2014.

See Table S4 for the change statistics of individual glaciers. The 28 August 2014 Landsat 8 image is used as background.

Table S5. Cumulative glacier area and number change for seven size classes in the Greater Caucasus in 1960,1986 and 2014.

\begin{tabular}{|r|r|r|r|r|r|r|}
\hline Size class & \multicolumn{3}{|c|}{ Area } & \multicolumn{3}{c|}{ Number } \\
\cline { 2 - 7 }$\left(\mathrm{km}^{2}\right)$ & $\mathbf{1 9 6 0}$ & $\mathbf{1 9 8 6}$ & $\mathbf{2 0 1 4}$ & $\mathbf{1 9 6 0}$ & $\mathbf{1 9 8 6}$ & $\mathbf{2 0 1 4}$ \\
\hline $0.01-0.05$ & 14.95 & 12.26 & 16.21 & 431 & 364 & 516 \\
\hline $0.05-0.1$ & 33.70 & 38.00 & 28.43 & 427 & 502 & 388 \\
\hline $0.1-0.5$ & 219.18 & 194.42 & 154.09 & 918 & 839 & 695 \\
\hline $0.5-1.0$ & 173.27 & 153.38 & 127.00 & 241 & 209 & 175 \\
\hline $1.0-5.0$ & 555.28 & 514.46 & 415.29 & 275 & 246 & 204 \\
\hline $5.0-10.0$ & 242.12 & 208.08 & 210.29 & 35 & 29 & 29 \\
\hline$>10.0$ & 436.28 & 361.68 & 241.72 & 22 & 20 & 13 \\
\hline
\end{tabular}




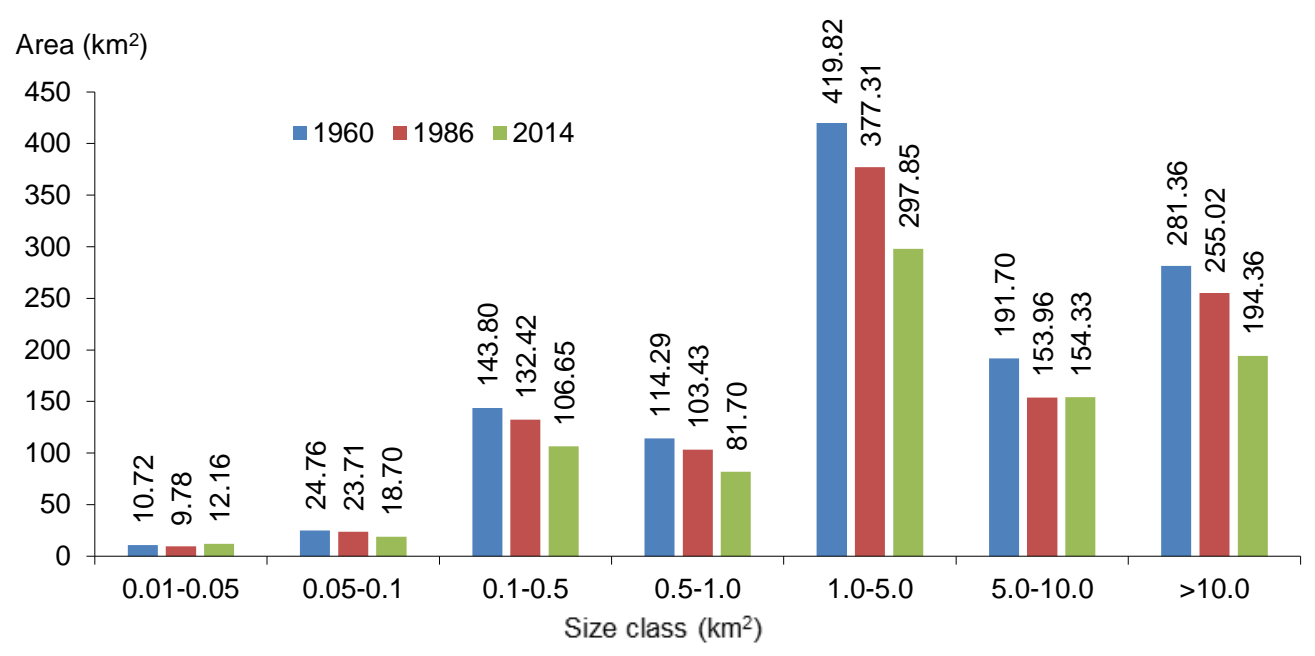

Figure S3. Cumulative glacier area values for seven size classes in 1960, 1986 and 2014 for the northern Greater Caucasus.

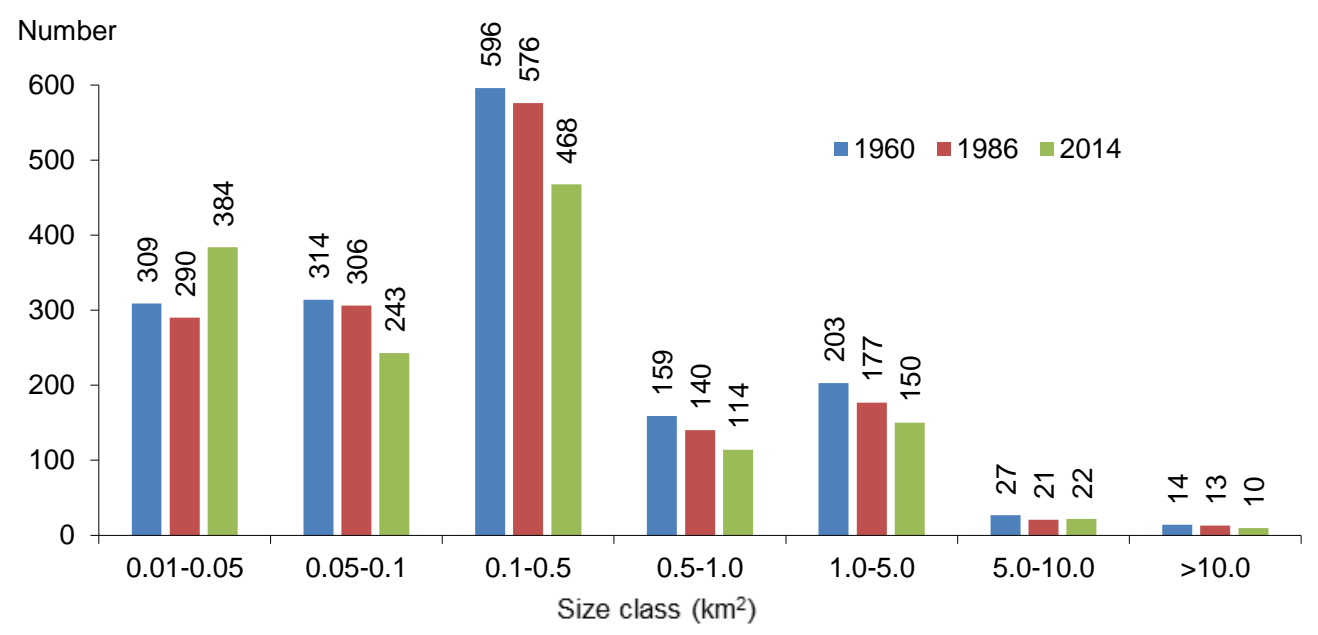

Figure S4. Cumulative glacier number values for seven size classes in 1960, 1986 and 2014 for the northern Greater Caucasus. 


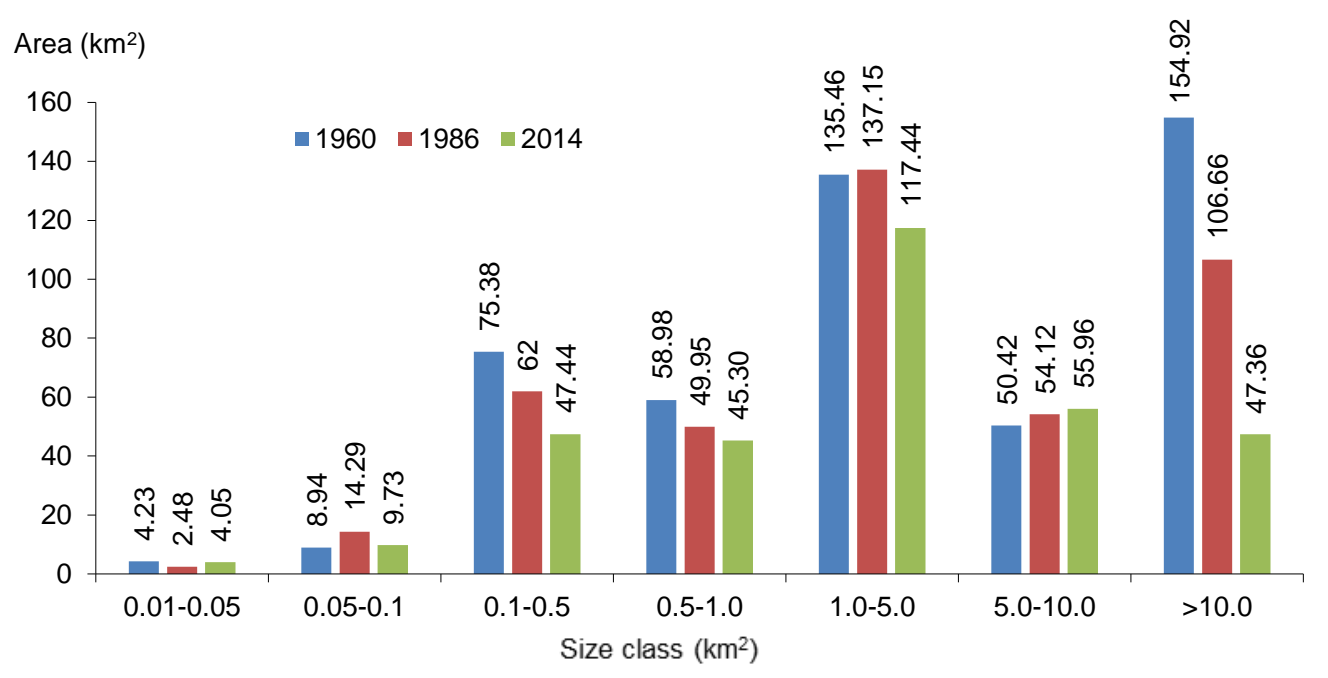

Figure S5. Cumulative glacier area values for seven size classes in 1960, 1986 and 2014 for the southern Greater Caucasus.

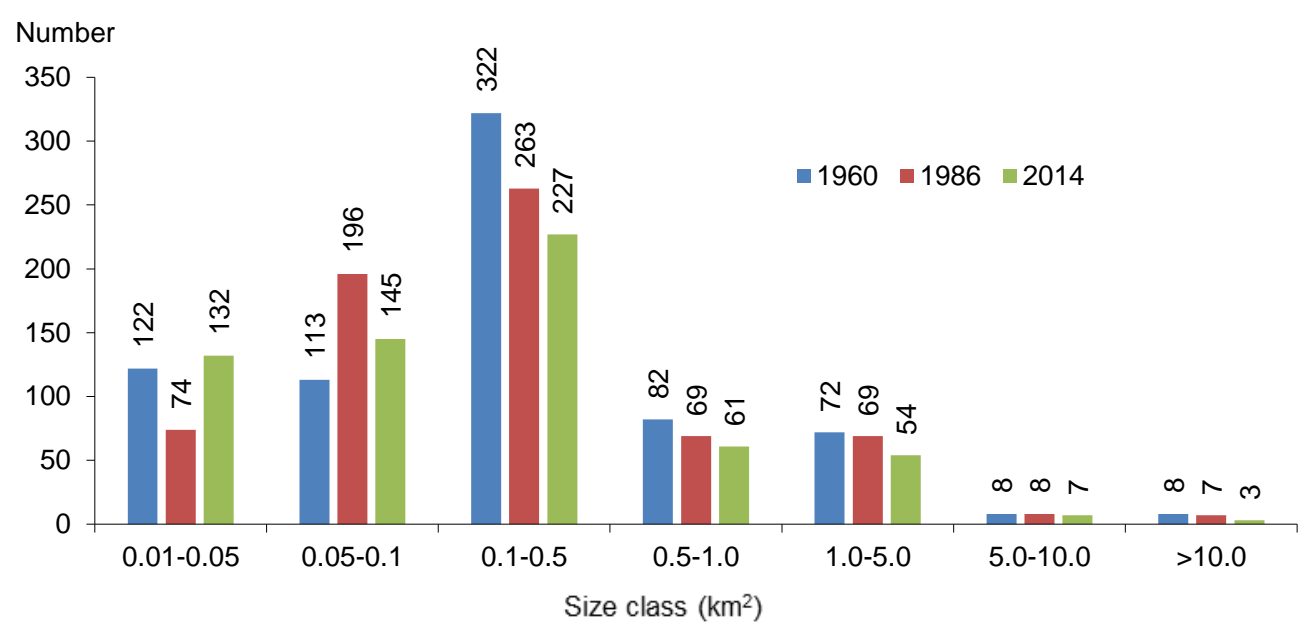

Figure S6. Cumulative glacier number values for seven size classes in 1960, 1986 and 2014 for the southern Greater Caucasus. 


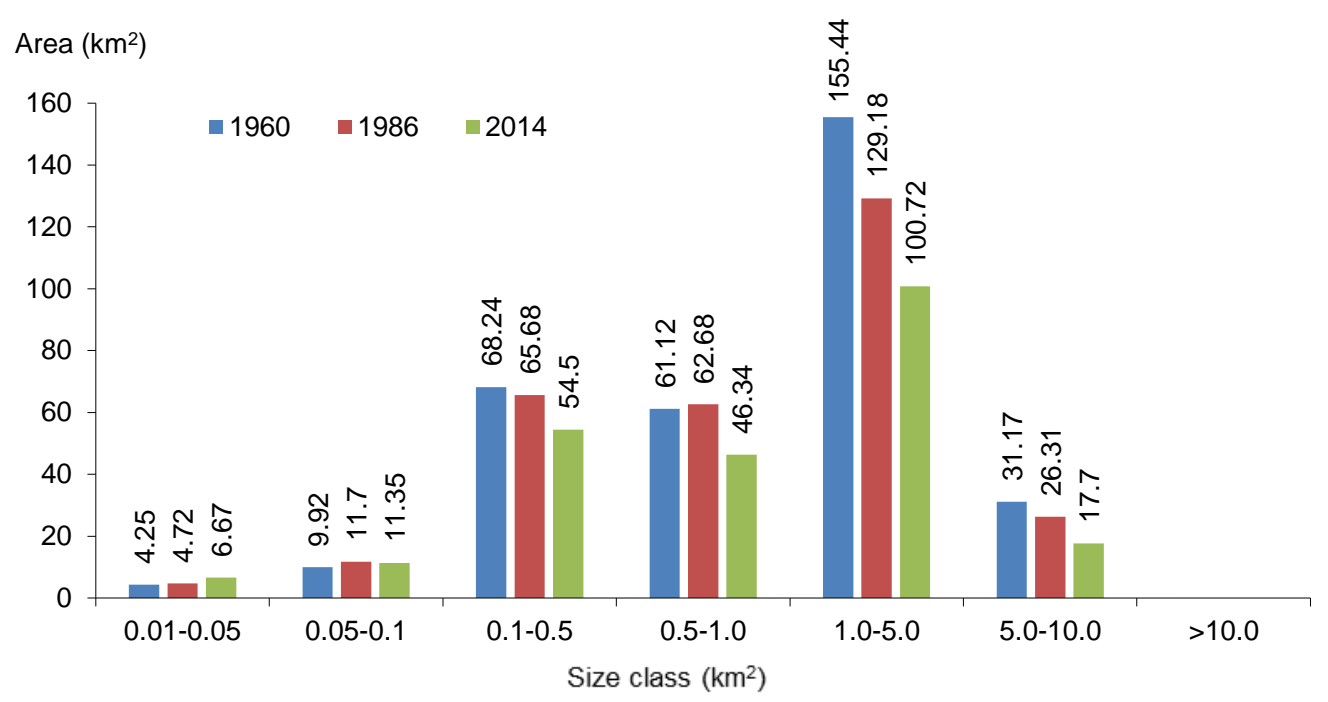

Figure S7. Cumulative glacier area values for seven size classes in 1960, 1986 and 2014 for the western Greater Caucasus.

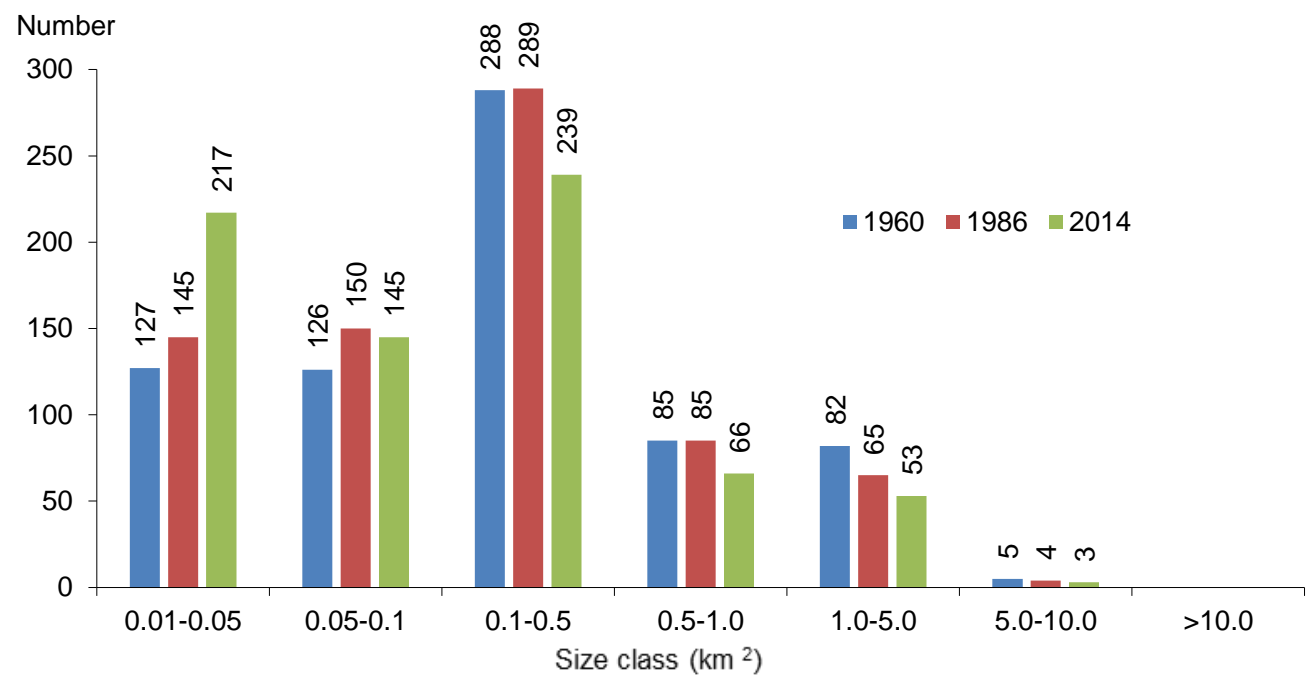

Figure S8. Cumulative glacier number values for seven size classes in 1960, 1986 and 2014 for the western Greater Caucasus. 


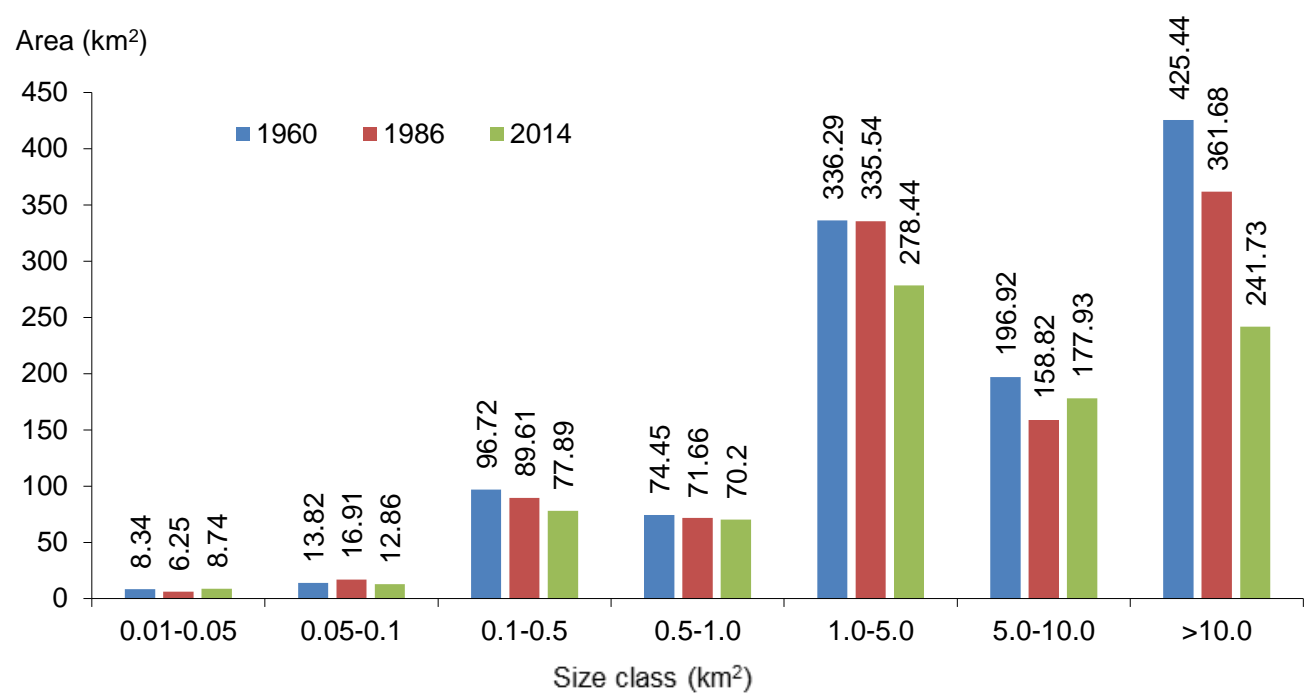

Figure S9. Cumulative glacier area values for seven size classes in 1960, 1986 and 2014 for the central Greater Caucasus.

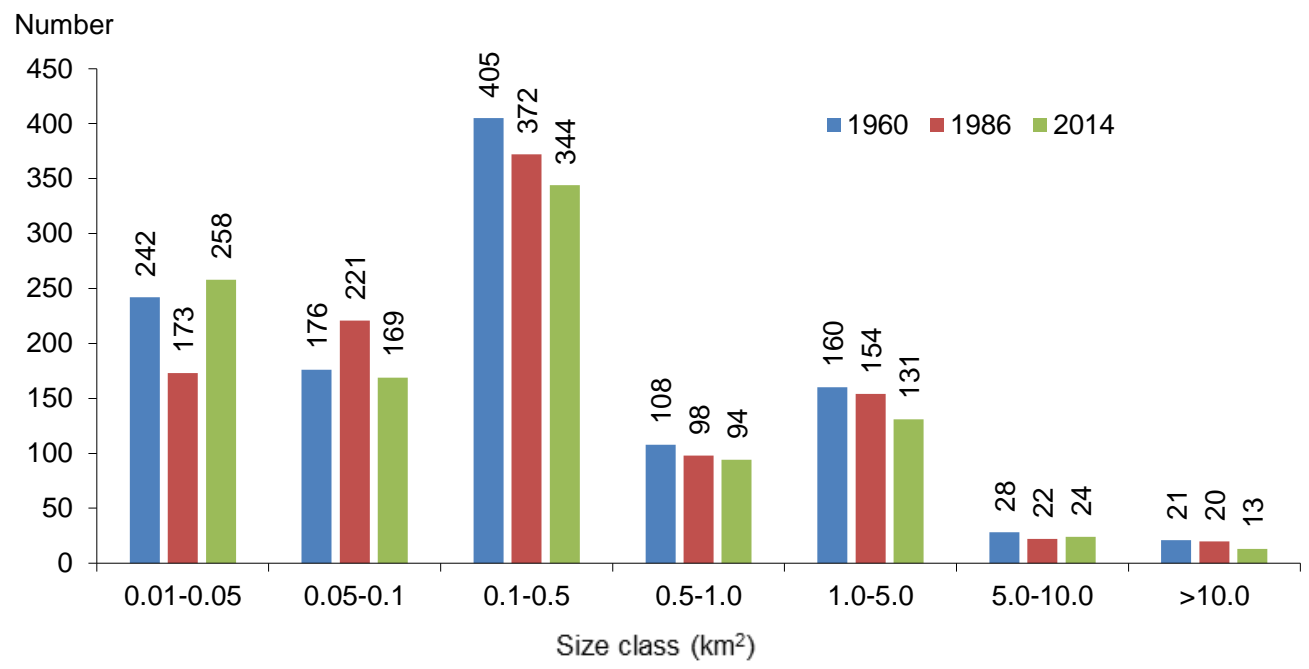

Figure S10. Cumulative glacier number values for seven size classes in 1960, 1986 and 2014 for the central Greater Caucasus. 


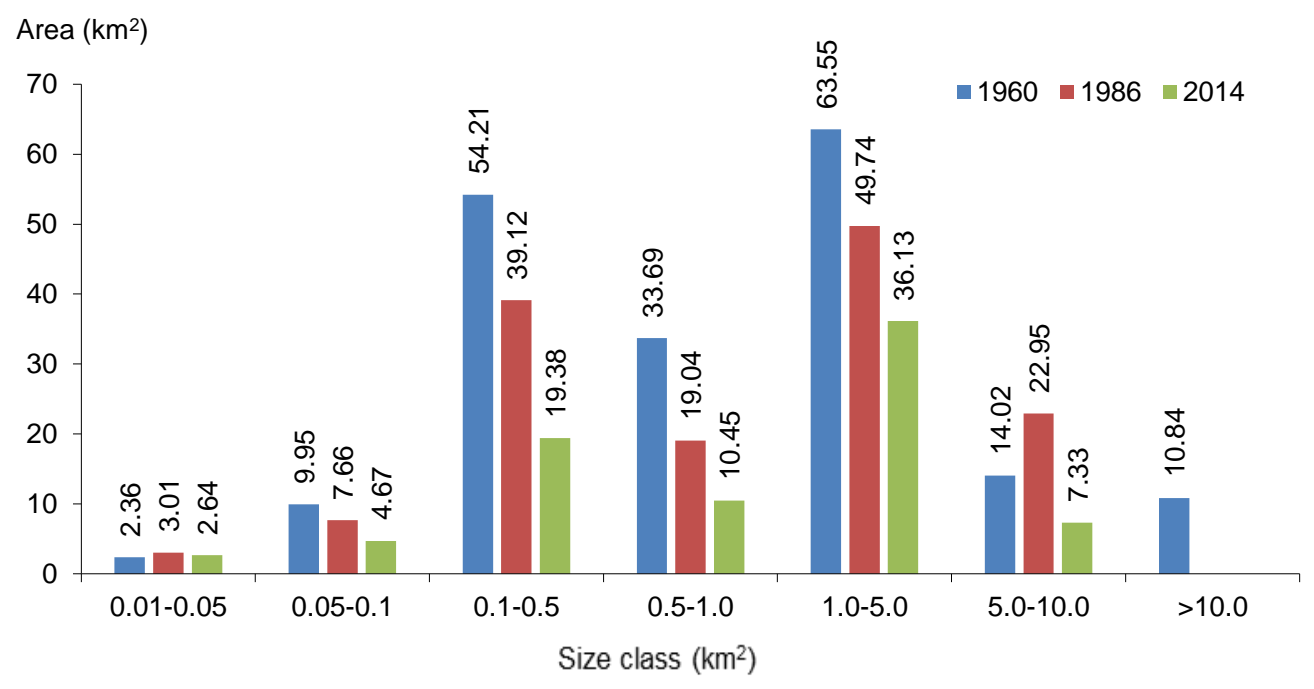

Figure S11. Cumulative glacier area values for seven size classes in 1960, 1986 and 2014 for the eastern Greater Caucasus.

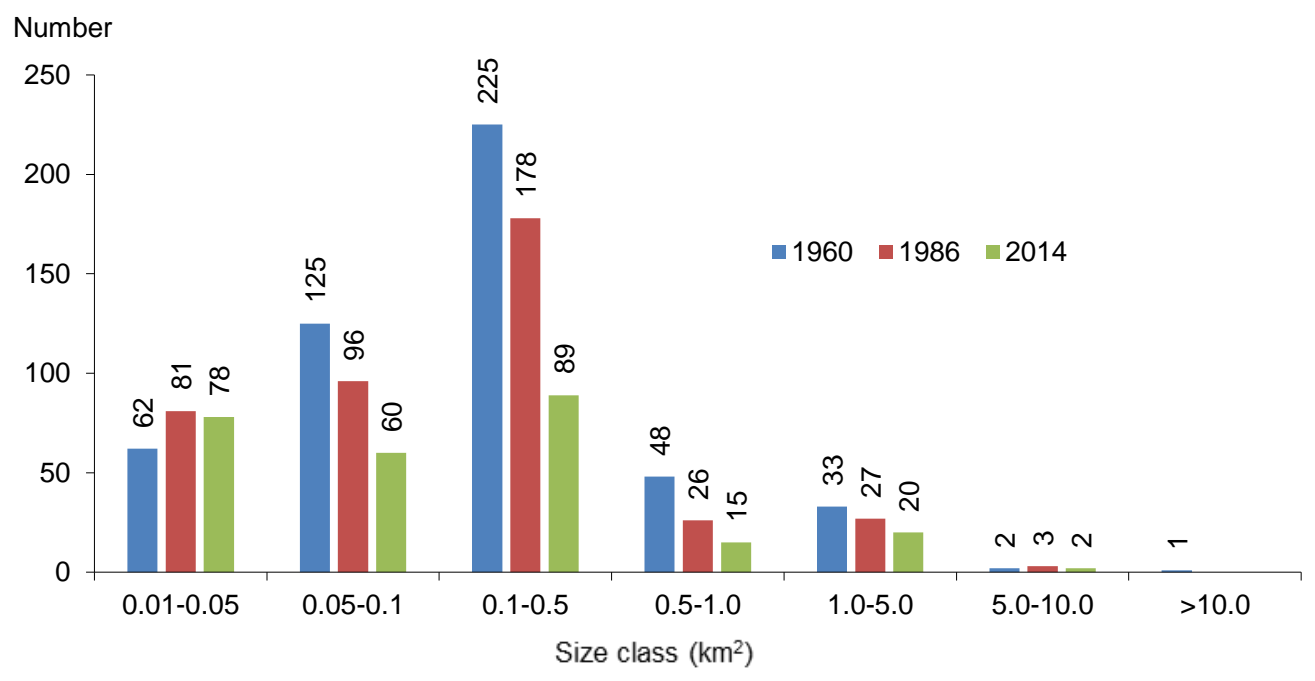

Figure S12. Cumulative glacier number values for seven size classes in 1960, 1986 and 2014 for the eastern Greater Caucasus. 


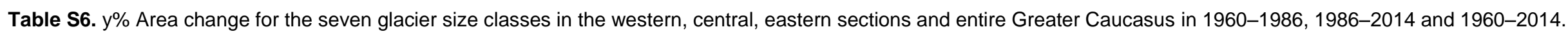

Western Caucasus

Eastern Caucasus

Entire Greater Caucasus

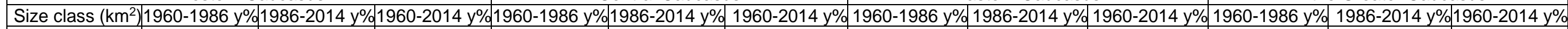

\begin{tabular}{|c|c|c|c|c|c|c|c|c|c|c|c|c|}
\hline $0.01-0.05$ & 0.42 & 1.47 & 1.05 & -0.96 & 1.42 & 0.08 & 1.05 & -0.43 & 0.21 & -0.69 & 1.15 & 0.15 \\
\hline $0.05-0.1$ & 0.69 & -0.1 & 0.26 & 0.85 & -0.85 & -0.12 & -0.88 & -1.39 & -0.98 & 0.49 & -0.89 & -0.28 \\
\hline $0.1-0.5$ & -0.77 & -0.6 & -0.37 & -0.28 & -0.46 & -0.36 & -1.07 & 1.8 & -1.18 & -0.43 & -0.74 & -0.55 \\
\hline $0.5-1.0$ & 0.09 & -0.93 & -0.44 & -0.14 & -0.07 & -0.1 & -1.67 & -1.61 & -1.27 & -0.44 & -0.61 & -0.49 \\
\hline $1.0-5.0$ & -0.65 & -0.78 & -0.65 & -0.008 & -0.6 & -0.31 & -0.83 & -0.98 & -0.8 & -0.28 & -0.68 & -0.46 \\
\hline $5.0-10.0$ & -0.59 & -1.16 & -0.8 & -0.74 & 0.43 & -0.17 & 2.45 & -2.43 & -0.88 & -0.54 & 0.03 & -0.24 \\
\hline$>10.0$ & - & - & - & -0.57 & -1.18 & -0.8 & - & - & - & -0.65 & -1.18 & -0.82 \\
\hline
\end{tabular}

Table S7. Characteristics of glaciers used for measuring length change. The average error terms are $\pm 15 \mathrm{~m}$.

\begin{tabular}{|c|c|c|c|c|c|c|c|c|c|c|c|c|c|}
\hline \multirow{2}{*}{ Name/WGI ID } & \multirow{2}{*}{ River basin } & \multirow{2}{*}{ Area 1960} & \multirow{2}{*}{ Area 1986} & \multirow{2}{*}{ Area 2014} & \multirow{2}{*}{$\begin{array}{c}1960- \\
1986 \\
\% \mathrm{yr}^{1}\end{array}$} & \multirow{2}{*}{$\begin{array}{c}1986- \\
2014 \\
\% \mathrm{yr}^{1}\end{array}$} & \multirow{2}{*}{$\begin{array}{c}1960- \\
2014 \\
\% \mathrm{yr}^{1}\end{array}$} & \multicolumn{2}{|c|}{$\begin{array}{c}\text { Length change } \\
1960-1986\end{array}$} & \multicolumn{2}{|c|}{$\begin{array}{c}\text { Length change } \\
\text { 1986-2014 }\end{array}$} & \multicolumn{2}{|c|}{$\begin{array}{c}\text { Length change } \\
\text { 1960-2014 }\end{array}$} \\
\hline & & & & & & & & $\mathrm{m}$ & $\mathrm{m} \mathrm{yr} \mathrm{r}^{1}$ & $\mathrm{~m}$ & $\mathrm{~m} \mathrm{yr} \mathrm{r}^{1}$ & $\mathrm{~m}$ & $\mathrm{~m} \mathrm{yr} r^{1}$ \\
\hline & & & & & & & & & & \multicolumn{4}{|c|}{ Glaciers with $>10 \mathrm{~km}^{2}$ area } \\
\hline Bezingi & Cherek-Bezingskiy & $40.42 \pm 0.98$ & $39.98 \pm 0.9$ & $37.47 \pm 0.94$ & -0.04 & -0.22 & -0.13 & -519 & -19.7 & -374 & -13.4 & -893 & -16.5 \\
\hline Dych-sy-Ailama & Cherek-Balkarskiy & $39.49 \pm 0.98$ & $34.85 \pm 0.94$ & $27.53 \pm 0.78$ & -0.45 & -0.75 & -0.56 & -461 & -17.7 & -1094 & -39.1 & -1555 & -28.8 \\
\hline Karaugom & Karaugom & $29.94 \pm 0.6$ & $29.17 \pm 0.62$ & $23.99 \pm 0.44$ & -0.09 & -0.63 & -0.36 & -164 & -6.3 & -1366 & -48.8 & -1530 & -28.3 \\
\hline Lekhziri & Enguri & $35.80 \pm 0.9$ & $33.95 \pm 0.94$ & $23.76 \pm 0.72$ & -0.19 & -1.07 & -0.62 & -859 & -33.0 & -736 & -26.3 & -1595 & -29.5 \\
\hline Agashtan & Cherek-Balkarskiy & $21.35 \pm 0.36$ & $20.39 \pm 0.32$ & $18.93 \pm 0.44$ & -0.17 & -0.25 & -0.20 & -368 & -14.2 & -587 & -21.0 & -955 & -17.7 \\
\hline Mizhirgi & Cherek-Bezingskiy & $13.77 \pm 0.5$ & $13.90 \pm 0.48$ & $12.71 \pm 0.48$ & +0.03 & -0.30 & -0.14 & -808 & -31.1 & +40 & +1.4 & -768 & -14.2 \\
\hline Tsaneri southern* & Enguri & $28.26 \pm 0.52$ & $14.38 \pm 0.32$ & $12.31 \pm 0.32$ & -0.21 & -0.51 & -1.04 & -448 & -17.2 & -781 & -27.9 & -1229 & -22.8 \\
\hline Tseya & Tseyadon & $14.03 \pm 0.42$ & $12.83 \pm 0.38$ & $11.87 \pm 0.36$ & -0.32 & -0.26 & -0.28 & -295 & -11.3 & -341 & -12.2 & -636 & -11.8 \\
\hline Tsaneri northern & Enguri & $--^{* \star}$ & $13.30 \pm 0.22$ & $11.28 \pm 0.22$ & -0.58 & -0.54 & - & - & - & -574 & -20.5 & - & - \\
\hline \multicolumn{14}{|c|}{ Glaciers with $5-10 \mathrm{~km}^{2}$ area } \\
\hline Kvitlodi & Enguri & $12.23 \pm 0.26$ & $11.65 \pm 0.24$ & $9.58 \pm 0.2$ & -0.18 & -0.63 & -0.40 & -598 & -23.0 & -883 & -31.5 & -1481 & -27.4 \\
\hline Adishi & Enguri & $10.48 \pm 0.22$ & $10.34 \pm 0.2$ & $9.58 \pm 0.2$ & -0.05 & -0.26 & -0.15 & -124 & -4.8 & -390 & -13.9 & -514 & -9.5 \\
\hline Challaati & Enguri & $12.71 \pm 0.36$ & $12.36 \pm 0.38$ & $9.24 \pm 0.28$ & -0.10 & -0.90 & -0.50 & -460 & -17.7 & -223 & -8.0 & -683 & -12.6 \\
\hline Khalde & Enguri & $11.87 \pm 0.38$ & $10.65 \pm 0.36$ & $8.59 \pm 0.26$ & -0.39 & -0.69 & -0.51 & -130 & -5.0 & -130 & -4.6 & -260 & -4.8 \\
\hline
\end{tabular}




\begin{tabular}{|c|c|c|c|c|c|c|c|c|c|c|c|c|c|}
\hline Shkhelda & Baksan & $13.61 \pm 0.48$ & $12.50 \pm 0.50$ & $8.28 \pm 0.65$ & -0.31 & -1.20 & -0.72 & -950 & -36.5 & -480 & -17.1 & -1430 & -26.5 \\
\hline Bashil & Chegem & $8.16 \pm 0.19$ & $7.91 \pm 0.19$ & $7.34 \pm 0.19$ & -0.11 & -0.25 & -0.18 & -230 & -8.8 & -530 & -18.9 & -760 & -14.1 \\
\hline Dolra & Enguri & $7.95 \pm 0.21$ & $6.44 \pm 0.16$ & $5.36 \pm 0.12$ & -0.73 & -0.59 & -0.60 & -595 & -22.9 & -160 & -5.7 & -755 & -14.0 \\
\hline & & & & & & & & & & \multicolumn{4}{|c|}{ Glaciers with $1-5 \mathrm{~km}^{2}$ area } \\
\hline Boko & Rioni & $5.07 \pm 0.12$ & $4.71 \pm 0.12$ & $4.62 \pm 0.11$ & -0.27 & -0.07 & -0.16 & -287 & -11.0 & -436 & -15.6 & -723 & -13.4 \\
\hline Mostotsete & Urukh Headwaters & $4.27 \pm 0.14$ & $3.58 \pm 0.012$ & $3.23 \pm 0.13$ & -0.62 & -0.34 & -0.45 & -105 & -4.0 & -135 & 4.8 & -240 & -4.4 \\
\hline Marukh northern & Malii Zelenchuk & $3.25 \pm 0.08$ & $3.30 \pm 0.08$ & $2.82 \pm 0.07$ & +0.05 & -0.51 & -0.24 & -255 & -9.8 & -240 & -8.6 & -495 & -9.2 \\
\hline Chungurjar & Ullukam & $3.13 \pm 0.09$ & $2.11 \pm 0.07$ & $1.88 \pm 0.07$ & -1.25 & -0.39 & -0.74 & -490 & -18.8 & -405 & -14.4 & -895 & -16.6 \\
\hline Tbilisa & Riorni & $2.90 \pm 0.10$ & $2.21 \pm 0.09$ & $1.91 \pm 0.08$ & -0.91 & -0.48 & -0.63 & -186 & 7.2 & -354 & -12.6 & -540 & -10.0 \\
\hline Sakeni & Kodori & $2.47 \pm 0.07$ & $2.39 \pm 0.08$ & $1.99 \pm 0.05$ & -0.12 & -0.59 & -0.35 & -560 & -21.5 & -275 & -9.8 & -835 & -15.5 \\
\hline Abano & Tergi (Terek) & $1.96 \pm 0.09$ & $1.49 \pm 0.09$ & $1.33 \pm 0.09$ & -0.92 & -0.38 & -0.60 & -550 & -21.2 & -240 & -8.6 & -790 & -14.6 \\
\hline & & & & & & & & & & \multicolumn{4}{|c|}{ Glaciers with $<1 \mathbf{~ k m}^{2}$ area } \\
\hline SU5T09106388 & Rioni & $0.86 \pm 0.05$ & $0.73 \pm 0.04$ & $0.69 \pm 0.03$ & -0.58 & -0.20 & -0.36 & -360 & -13.8 & -70 & -2.5 & -430 & -8.0 \\
\hline *** & Sharo Argun & $0.90 \pm 0.05$ & $0.77 \pm 0.04$ & $0.55 \pm 0.03$ & -0.55 & -1.02 & -0.72 & -65 & -2.5 & -270 & -9.6 & -335 & -6.2 \\
\hline SU4G08011072 & Tergi (Terek) & $0.62 \pm 0.03$ & $0.55 \pm 0.03$ & $0.42 \pm 0.02$ & -0.43 & -0.84 & -0.60 & -60 & -2.3 & -310 & -11.1 & -370 & -6.9 \\
\hline *** & Andiiskoe Koisu & $0.63 \pm 0.04$ & $0.43 \pm 0.03$ & $0.29 \pm 0.02$ & -1.22 & -1.16 & -0.99 & -243 & -9.3 & -245 & -8.8 & -588 & -10.9 \\
\hline SU4G08007139 & Cherek-Balkarskiy & $0.36 \pm 0.03$ & $0.37 \pm 0.03$ & $0.26 \pm 0.02$ & +0.10 & -1.06 & -0.51 & -189 & -7.3 & -210 & -7.5 & -399 & -7.4 \\
\hline SU4G08011083 & Tergi (Terek) & $0.99 \pm 0.04$ & $0.55 \pm 0.03$ & $0.15 \pm 0.01$ & -1.70 & -2.59 & -1.57 & -234 & -9.0 & -470 & -16.8 & -704 & -13.0 \\
\hline SU5T09105282 & Enguri & $0.19 \pm 0.02$ & $0.13 \pm 0.01$ & $0.10 \pm 0.005$ & -1.21 & -0.82 & -0.87 & -60 & -2.3 & -60 & -2.1 & -120 & -2.2 \\
\hline
\end{tabular}

${ }^{*},{ }^{* *}$ Until the 1980s the Southern and Northern Tsaneri were merged as one compound-valley type glacier. Their division likely happened in $1980-1985$.

*** Omitted in WGI database. 


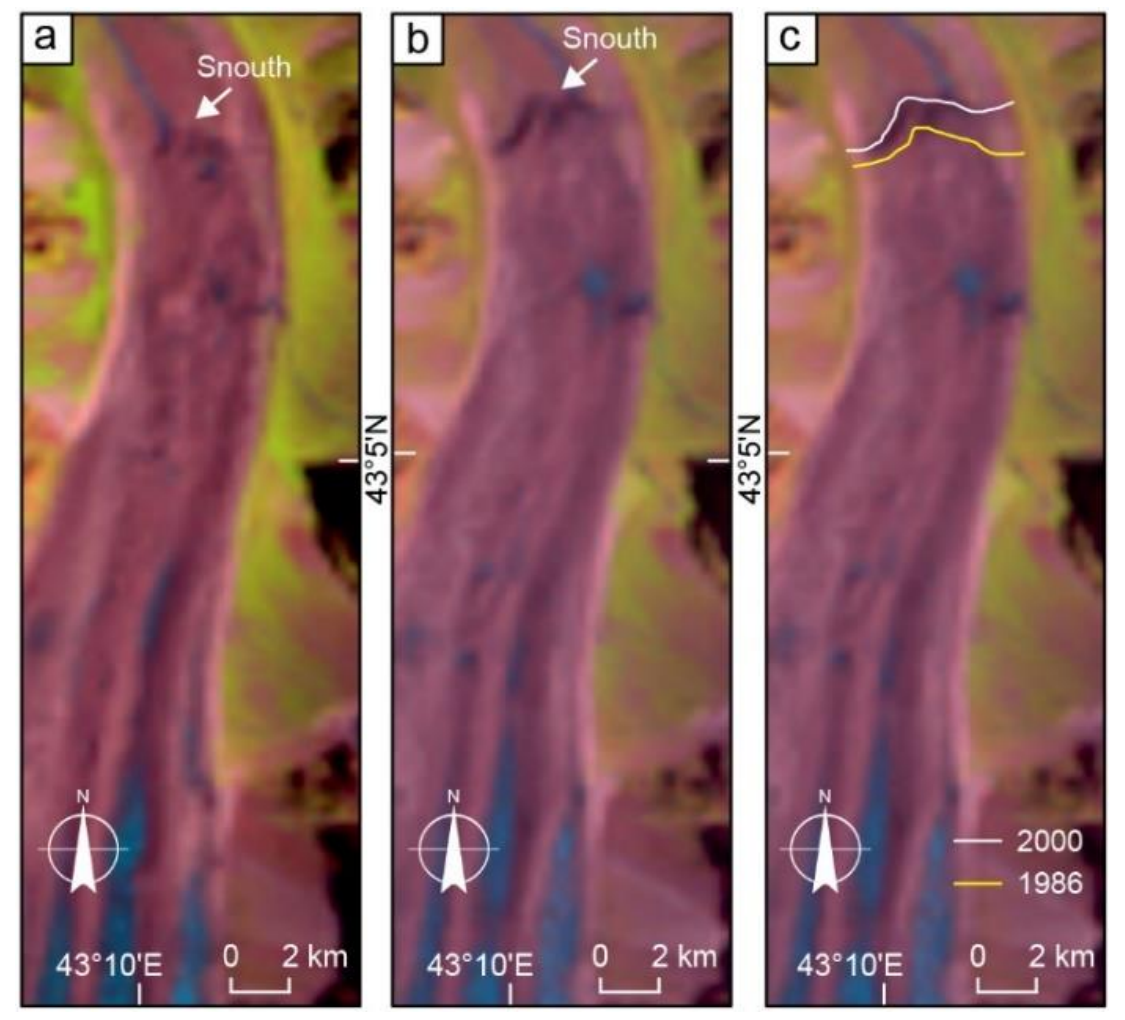

Figure S13. Mizhirgi Glacier advance between 1986-2000. (a) Landsat 5 TM, 6 August 1986. (b) Landsat 5 TM, 12 August 2000. In 1986, the meltwater flow comes from a different position at the terminus. (c) With the snout comparison, it is visible that the snout has advanced. 


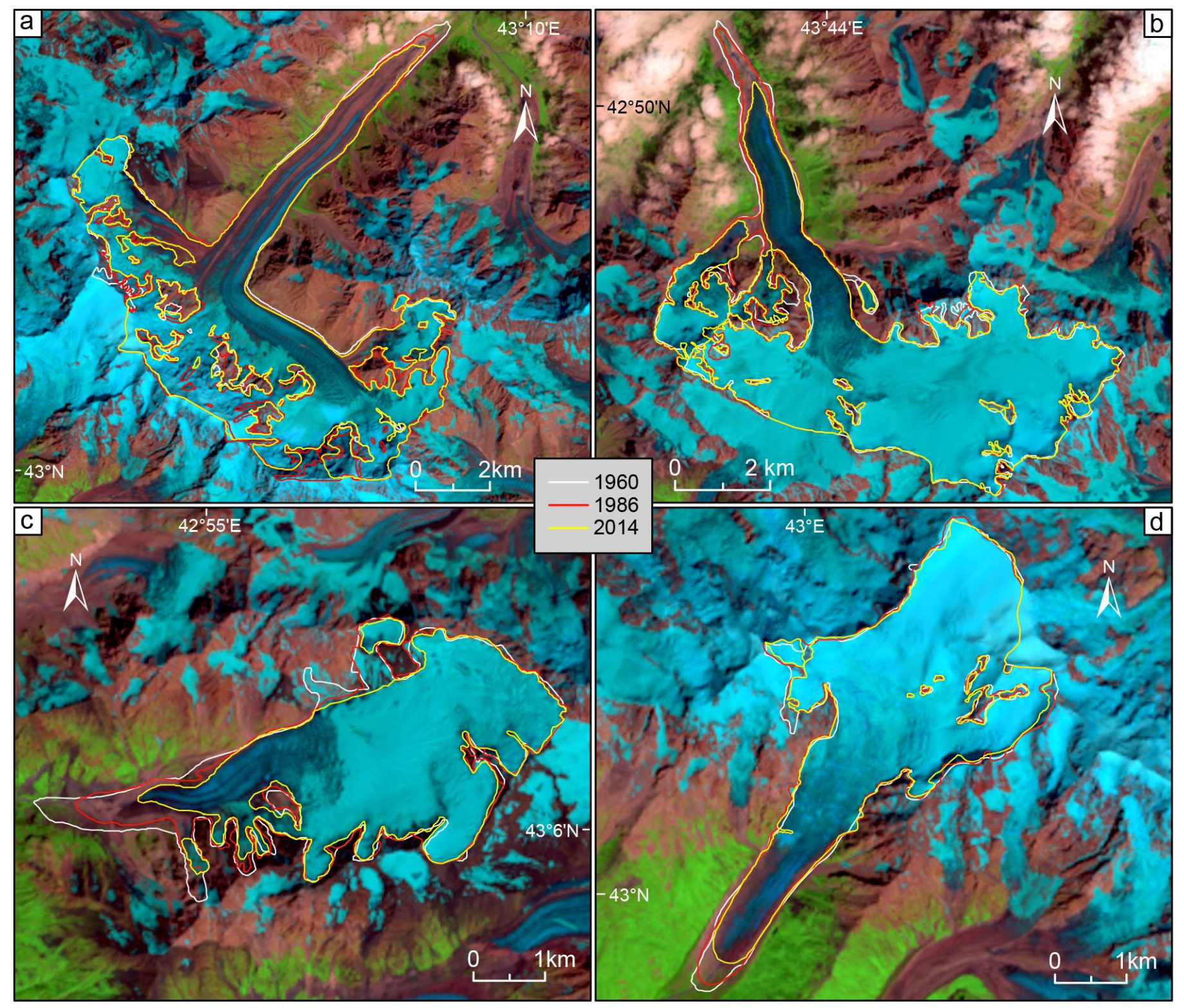

Figure S14. (a) Bezingi, (b) Karaugom, (c) Kvitlodi and (d) Adishi glaciers reduction in the years 1960, 1986 and 2014. The 03 August 2014 Landsat 8 image is used as background. 